\title{
Viscoelastic effects on drop deformation in steady shear
}

\author{
By PENGTAO YUE ${ }^{1}$, JAMES J. FENG ${ }^{1}$, \\ CHUN LIU ${ }^{2}$ AND JIE SHEN S $^{3}$ \\ ${ }^{1}$ Department of Chemical and Biological Engineering and Department of Mathematics, \\ University of British Columbia, Vancouver, BC V6T 1Z4, Canada \\ ${ }^{2}$ Department of Mathematics, The Pennsylvania State University, University Park, PA 16802, USA \\ ${ }^{3}$ Department of Mathematics, Purdue University, West Lafayette, IN 47907, USA
}

(Received 1 February 2005 and in revised form 24 May 2005)

This paper applies a diffuse-interface model to simulate the deformation of single drops in steady shear flows when one of the components is viscoelastic, represented by an Oldroyd-B model. In Newtonian fluids, drop deformation is dominated by the competition between interfacial tension and viscous forces due to flow. A fundamental question is how viscoelasticity in the drop or matrix phase influences drop deformation in shear. To answer this question, one has to deal with the dual complexity of nonNewtonian rheology and interfacial dynamics. Recently, we developed a diffuse-interface formulation that incorporates complex rheology and interfacial dynamics in a unified framework. Using a two-dimensional spectral implementation, our simulations show that, in agreement with observations, a viscoelastic drop deforms less than a comparable Newtonian drop. When the matrix is viscoelastic, however, the drop deformation is suppressed when the Deborah number $D e$ is small, but increases with $D e$ for larger $D e$. This non-monotonic dependence on matrix viscoelasticity resolves an apparent contradiction in previous experiments. By analysing the flow and stress fields near the interface, we trace the effects to the normal stress in the viscoelastic phase and its modification of the flow field. These results, along with prior experimental observations, form a coherent picture of viscoelastic effects on steady-state drop deformation in shear.

\section{Introduction}

Flow-induced drop deformation has long fascinated fluid dynamicists (Taylor 1932). Using experimental observations, asymptotic analysis and numerical calculations, researchers have constructed a reasonably complete picture for Newtonian drops deforming in a Newtonian medium (Rallison 1984; Stone 1994).

Two-phase complex fluids such as emulsions and blends often involve components that are microstructured complex fluids themselves. As drop dynamics is the key to understanding interfacial morphology in two-phase materials, a fundamental question arises about the effect of the component rheology on the deformation of drops. A particularly important example is the effect of viscoelasticity on drop deformation in polymer blends (Tucker \& Moldenaers 2002). Previous efforts on this problem have produced a sizable literature, but the picture is incomplete in so far as certain prior results seemingly contradict each other, and the physical mechanisms behind the observed behaviour remain obscure.

Early insights have come from heuristic ideas that the drop shape is determined by the balance among viscous and elastic forces inside and outside the drop and the 
interfacial tension. Vanoene (1972) defined a dynamic interfacial tension which includes the difference of the normal stresses between the two phases. Similar force balances were considered by Levitt, Macosko \& Pearson (1996). Such reasoning predicts that the normal stresses of the drop phase resist drop deformation, while those of the matrix enhance deformation. In extensional flows, this heuristic argument has largely been validated by more rigorous phenomenological models (Maffettone \& Greco 2004; Yu, Zhou \& Bousmina 2005), numerical simulations (Ramaswamy \& Leal 1999a,b; Hooper et al. 2001) and experiments (Mighri, Ajji \& Carreau 1997; Tretheway \& Leal 2001). However, Ramaswamy \& Leal (1999a) pointed out the naïveté of the heuristic argument: the viscoelastic effect comprises not only the direct contribution of the viscoelastic stresses at the interface, but also modification of the flow by the stresses over the entire domain.

In steady shear flows, the situation is not as clear-cut as in extensional flows. If the drop phase is viscoelastic, there is again consensus that it inhibits deformation. This effect has been observed experimentally by Elmendorp \& Maalcke (1985), Mighri, Carreau \& Ajji (1998) and Lerdwijitjarud, Sirivat \& Larson (2004), and predicted by Pillapakkam \& Singh (2001) and Maffettone \& Greco (2004). When the suspending fluid is viscoelastic, however, there is contradiction among the experiments. Elmendorp \& Maalcke (1985), using shear-thinning polymer solutions as the matrix, observed that increasing the normal stress in the matrix increases the deformation of a Newtonian drop. Mighri et al. (1998) reached the same conclusion by using Boger fluids. Guido, Simeone \& Greco (2003), on the other hand, presented clear evidence that a Newtonian drop deforms less if the matrix is viscoelastic. Flumerfelt (1972) reported that a viscoelastic matrix stabilizes a Newtonian drop against breakup, in indirect support of Guido et al. (2003).

A handful of theoretical studies have considered drop deformation in a viscoelastic medium undergoing shear (Khismatullin, Renardy \& Renardy 2005). But none directly addresses the contradictory observations, let alone resolves the puzzle. Greco (2002) obtained a perturbation solution for drop deformation in a second-order fluid. Viscoelasticity turns out to have no effect on drop deformation at the leading order. Thus, the opposing trends observed in experiments must be due to finite viscoelastic effects, on which the perturbation solution can shed little light. Maffettone \& Greco (2004) and Yu et al. (2005) predicted reduced drop deformation, in agreement with the experiment of Guido et al. (2003) and contradicting those of Elmendorp \& Maalcke (1985) and Mighri et al. (1998). Because of the phenomenological nature of these models, however, they do not provide any insight into the underlying physics.

Our objective in this work is to use numerical simulations and detailed analysis of the underlying physics to clear up the confusion in the literature and construct a coherent picture for shear-induced drop deformation. This is made possible by a recently developed diffuse-interface model for two-phase flows of complex fluids, which deals with the interfacial dynamics and non-Newtonian rheology in a unified framework (Yue et al. 2004). So far, we have applied this formalism to drop coalescence and retraction in Oldroyd-B viscoelastic fluids and liquid crystals. The former will be relevant to this work.

\section{Theoretical and numerical models}

Our model differs from other fixed-grid methods in that the interface is treated as physically diffuse. The interfacial position and thickness are determined by a phasefield variable $\phi$ whose evolution is governed by Cahn-Hilliard dynamics. In this 
way, the structure of the interface is rooted in molecular forces; the tendencies for mixing and demixing are balanced through the non-local mixing energy. Owing to its energy-based formulation, the model allows easy incorporation of complex rheology. The free energy describing the conformation of the microstructure is simply added to the mixing energy to form the total free energy. Then a formal variational procedure applied to the total free energy gives rise to the proper constitutive equation for the microstructured fluids in addition to the evolution equation for $\phi$. Yue et al. (2004) have given a detailed derivation of the theoretical model and described the numerical scheme using spectral discretization. Feng et al. (2005) have discussed the advantages and challenges of this approach in the general context of moving-interface problems. Here, we will specialize the formalism for a mixture of a Newtonian and an Oldroyd-B fluid, and summarize the main features of the numerical procedure.

The Newtonian and Oldroyd-B components are immiscible except in a very thin interfacial region. The phase-field variable $\phi$ is such that the concentrations of the Oldroyd-B and Newtonian components are $(1+\phi) / 2$ and $(1-\phi) / 2$, respectively. For the mixing energy, we adopt the familiar Ginzburg-Landau form:

$$
f_{\text {mix }}(\phi, \nabla \phi)=\frac{1}{2} \lambda|\nabla \phi|^{2}+\frac{\lambda}{4 \epsilon^{2}}\left(\phi^{2}-1\right)^{2},
$$

where $\lambda$ is the energy density and $\epsilon$ is a capillary width that scales with the thickness of the diffuse interface. As $\epsilon \rightarrow 0$, the ratio $\lambda / \epsilon$ produces the interfacial tension in the classical sense (Jacqmin 1999; Yue et al. 2004).

An Oldroyd-B fluid consists of a dilute suspension of linear Hookean dumbbells in a Newtonian solvent (Bird et al. 1987). If the dumbbells have an elastic constant $H$, a number density $n$ and a configuration distribution $\Psi(\boldsymbol{Q})$ for the connector $\boldsymbol{Q}$, we write the dumbbell elastic energy as

$$
f_{d}=n \int_{R^{3}}\left(k T \ln \Psi+\frac{1}{2} H \boldsymbol{Q} \cdot \boldsymbol{Q}\right) \Psi \mathrm{d} Q,
$$

where $k$ is the Boltzmann constant and $T$ is the temperature. Now the total free energy density of the two-phase system is $f=f_{\text {mix }}+(1+\phi) f_{d} / 2$.

Through a variational procedure detailed by Yue et al. (2004), an elastic stress tensor can be derived from the total free energy. Adding the proper viscous stress, we write the total stress tensor as

$$
\boldsymbol{\tau}=\left(\frac{1-\phi}{2} \mu_{n}+\frac{1+\phi}{2} \mu_{s}\right)\left[\nabla v+(\nabla v)^{T}\right]+\boldsymbol{\tau}_{p}-\lambda \nabla \phi \otimes \nabla \phi,
$$

where $\mu_{n}$ is the viscosity of the Newtonian component and $\mu_{s}$ is the viscosity of the Newtonian solvent in the Oldroyd-B fluid. The dumbbell elastic stress $\tau_{p}=(1+\phi) \tau_{d} / 2$ with $\boldsymbol{\tau}_{d}=-n k T \boldsymbol{I}+n H\langle\boldsymbol{Q} \boldsymbol{Q}\rangle$ being exactly the Kramers expression for the dumbbell stress (Bird et al. 1987). Here $\langle\cdot\rangle$ indicates the average of a quantity over all dumbbell configurations. $\boldsymbol{\tau}_{d}$ obeys the Maxwell equation:

$$
\boldsymbol{\tau}_{d}+\lambda_{H} \boldsymbol{\tau}_{d(1)}=\mu_{p}\left[\nabla \boldsymbol{v}+(\nabla \boldsymbol{v})^{T}\right]
$$

where the subscript (1) denotes the upper convected derivative. $\lambda_{H}=\zeta /(4 H)$ is the relaxation time, $\zeta$ being the friction coefficient, and $\mu_{p}=n k T \lambda_{H}$ is the polymer viscosity. The evolution of $\phi$ is governed by the Cahn-Hilliard equation with mobility $\gamma$ :

$$
\frac{\partial \phi}{\partial t}+\boldsymbol{v} \cdot \nabla \phi=\gamma \lambda \nabla^{2}\left[-\nabla^{2} \phi+\frac{\phi\left(\phi^{2}-1\right)}{\epsilon^{2}}\right] .
$$



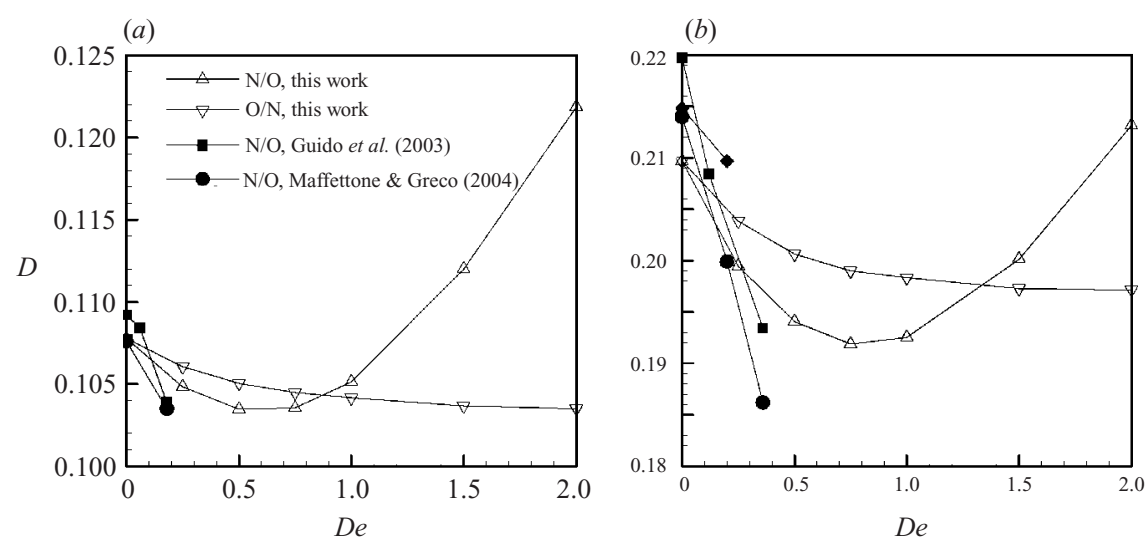

FIGURE 1. Steady-state deformation parameter $D$ as a function of the Deborah number $D e$ for two capillary numbers: ( $a$ ) $C a=0.1,(b) C a=0.2$. Experimental data of Guido et al. (2003) and predictions by Maffettone \& Greco (2004) are also shown. Comparison with these and other previous studies will be discussed at the end.

Equations (2.3), (2.4) and (2.5), along with the continuity and momentum equations $\nabla \cdot \boldsymbol{v}=0$ and $\nabla p=\nabla \cdot \boldsymbol{\tau}$, form the governing equations for our two-phase system. Inertia is neglected as the complex fluids are typically highly viscous. We will also refer to a dimensionless 'configuration tensor' $\boldsymbol{A}=(H / k T)\langle\boldsymbol{Q} \boldsymbol{Q}\rangle=\left(\lambda_{H} / \mu_{p}\right) \boldsymbol{\tau}_{d}+\boldsymbol{I}$ later in the discussion.

We will use the two-dimensional spectral method developed by Yue et al. (2004), with a Fourier or Chebyshev-Galerkin discretization on a regular grid depending on spatial periodicity. The number of operations per time step scales as $O(N \log N), N$ being the number of unknowns. We have carried out grid and time-step refinements to establish the adequacy of our spatial and temporal resolutions. The interface typically requires $7-10$ grids to resolve, and the total number of grid points is typically $2048 \times 1024$. In all cases tested, the temporal resolution is adequate as long as the simulation is stable.

\section{Steady-state drop deformation}

The drop deformation can be represented by $D=(L-B) /(L+B), L$ and $B$ being the longest and shortest lengths from the centre to the surface. For a Newtonian drop in a Newtonian matrix, $D$ increases with the capillary number $C a=\mu \dot{\gamma} a / \sigma$, where $\mu$ is the matrix viscosity, $\dot{\gamma}$ is the shear rate, $a$ is the undeformed drop radius and $\sigma$ is the interfacial tension. The $D \sim C a$ curve is well known, and Yue et al. (2004) used it to validate our numerical code. To explore the effects of viscoelasticity on $D$, we have simulated three cases: a Newtonian drop in a Newtonian matrix $(\mathrm{N} / \mathrm{N})$, a Newtonian drop in an Oldroyd-B matrix (N/O) and an Oldroyd-B drop in a Newtonian matrix $(\mathrm{O} / \mathrm{N})$. In each case, we have used equal viscosity between the two components; for $\mathrm{N} / \mathrm{O}$ and $\mathrm{O} / \mathrm{N}, \mu_{n}=\mu_{p}+\mu_{s}$ with $\mu_{p}=\mu_{s}$. The magnitude of viscoelasticity is represented by the Deborah number $D e=\lambda_{H} \dot{\gamma}$.

The main results of this paper are shown in figure 1 , which plots $D$ as a function of $D e$ for two values of $C a$. For $\mathrm{O} / \mathrm{N}, D$ decreases monotonically with increasing $D e$. For $\mathrm{N} / \mathrm{O}$, on the other hand, the curve has a minimum. Thus, viscoelasticity in the matrix suppresses drop deformation for small $D e$, in fact more strongly than in the $\mathrm{O} / \mathrm{N}$ case, but enhances drop deformation for large $D e$. To understand the origin of 


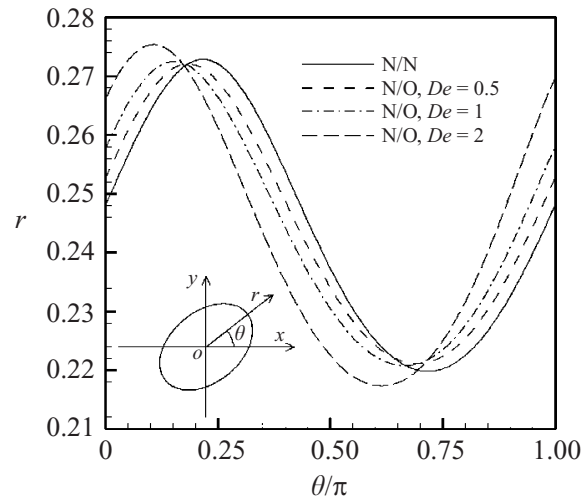

FIGURE 2. The drop surface in polar coordinates for $\mathrm{N} / \mathrm{O}$ at $C a=0.1$. Only half of the drop is shown.
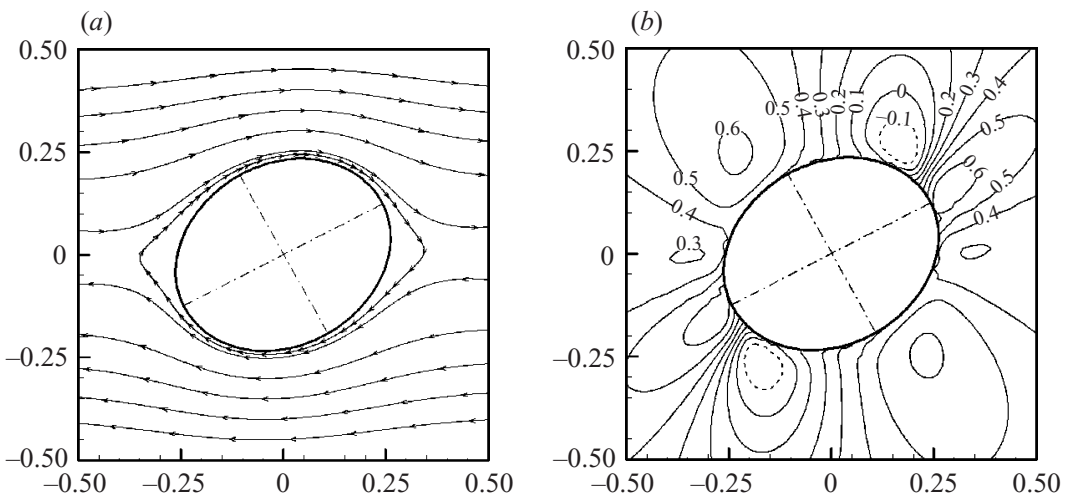

FiguRE 3. Flow kinematics in the viscoelastic matrix for $\mathrm{N} / \mathrm{O}$ at $C a=0.1$ and $D e=1$. (a) Streamlines. The dash-dot lines indicate the drop's equator and poles. $(b)$ Extensional rate along the predominant orientation of the dumbbells $\dot{\epsilon}_{d}=\boldsymbol{m} \cdot \nabla \boldsymbol{v} \cdot \boldsymbol{m}$, where $\boldsymbol{m}$ is a unit vector along the principal eigenvector of $\boldsymbol{A}$.

this surprising behaviour, we will analyse the flow and stress fields around the drop at several $D e$ and a fixed $C a=0.1$.

\subsection{A Newtonian drop in an Oldroyd-B matrix $(N / O)$}

To facilitate analysis of the flow and stress fields near the interface, we represent the drop shape by the $r(\theta)$ plot in figure 2, where $\theta$ is measured from the direction of shear ( $x$-axis). As $D e$ increases from 0 to $2, L$ and $B$ vary non-monotonically in accordance with figure 1 . The location of maximum radius $L$ moves monotonically toward smaller $\theta$. So increasing viscoelasticity in the matrix aligns the drop toward the direction of shear. We will refer to the end of $L$ as the drop's 'pole' and the end of $B$ as its 'equator'.

Figure 3 illustrates the flow kinematics in the Oldroyd-B matrix for $D e=1$. In this case, the pole of the elongated drop is at $\theta \approx 0.15 \pi$ and its equator is at $\theta \approx 0.65 \pi$. The most important features of the flow, as far as the dumbbells are concerned, are the accelerating regions near the equator and the decelerating regions upstream of the poles. To anticipate the reaction of the dumbbells, we plot in figure $3(b)$ the extensional rate $\dot{\epsilon}_{d}$ along the predominant orientation of the dumbbells, namely along the principal eigenvector of the configuration tensor $\boldsymbol{A}$. As expected, $\dot{\epsilon}_{d}$ is maximum 

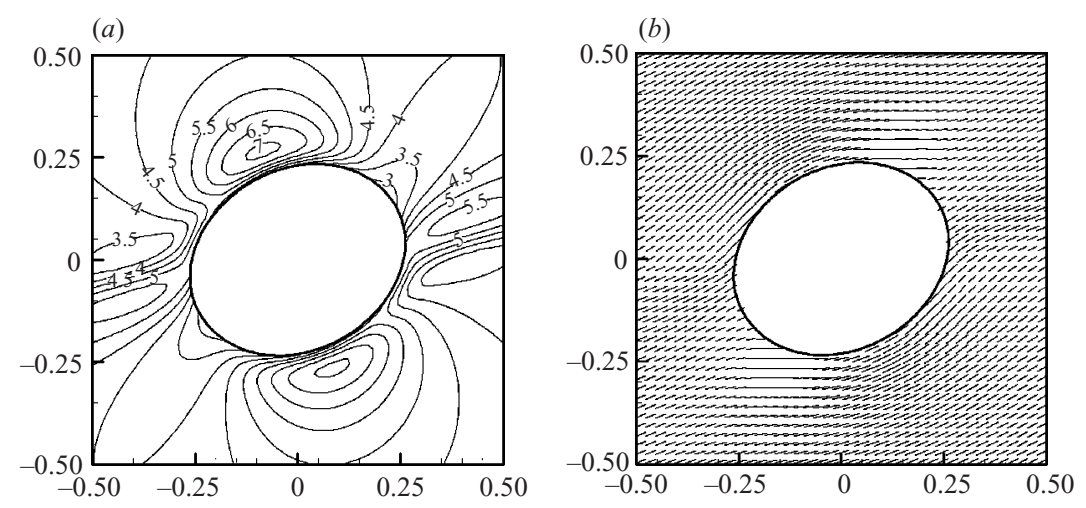

FIgURE 4. Configuration of dumbbells in the Oldroyd-B matrix for $\mathrm{N} / \mathrm{O}$ at $C a=0.1$ and $D e=1$. (a) Contours of $\operatorname{tr}(\boldsymbol{A})$, indicating the length of the dumbbells. (b) Orientation of dumbbells indicated by line segments along $\boldsymbol{m}$ with a length proportional to $\operatorname{tr}(\boldsymbol{A})$.

in the stretching region near the equator and minimum in the compressive region upstream of the poles.

Figure 4 shows the configuration of the dumbbells around the drop. At the poles, the dumbbells are nearly perpendicular to the interface. Thus, they will generate a tensile stress that stretches the drop at the poles, as for a drop in planar extensional flow (Ramaswamy \& Leal 1999b). Here, however, $\operatorname{tr}(\boldsymbol{A})$ is minimum at the poles, and the stretching effect is weak. At the equator, $\operatorname{tr}(\boldsymbol{A})$ is maximum and the dumbbell orientation $\boldsymbol{m}$ is tangential to the interface. The polymer produces a large tensile stress in this region, which, though not contributing directly to normal forces on the drop, does so indirectly as will be explained shortly. Comparing figures $3(b)$ and $4(a)$ shows that the maximum (or minimum) of $\operatorname{tr}(\boldsymbol{A})$ occurs some distance downstream of the maximum (or minimum) of $\dot{\epsilon}_{d}$. This simply reflects the finite relaxation time of the polymer molecules.

The qualitative features described above prevail for other $D e$ as well. Now we can examine the polymer and hydrodynamic contributions to the normal force on the outside of the drop. The flow inside the Newtonian drop is largely the same for $\mathrm{N} / \mathrm{N}$ and N/O. Figure 5 plots the distributions of various stress components along the 'outer edge' of the drop surface, taken here to be the level set of $\phi=0.9$. The polymer normal stress $\tau_{p n}$ is largest near the drop's pole and smallest near the equator (figure $5 a$ ). This is consistent with the orientation of dumbbells in figure $4(b)$. With increasing $D e$, the maximum of $\tau_{p n}$ increases in magnitude while its location shifts downstream toward smaller $\theta$. This is again the result of the increasing relaxation time $\lambda_{H}$. Thus, with increasing $D e, \tau_{p n}$ tends to align the drop with the flow direction and increase the stretch on the poles. Meanwhile, the compression on the equator of the drop decreases slightly.

The viscous normal stress $\tau_{s n}$ in figure $5(b)$ is comparable with $\tau_{p n}$ in magnitude. But with increasing $D e, \tau_{s n}$ is reduced near the poles, thus weakening the stretching of the drop there. On the equator, $\tau_{s n}$ also weakens its compression. This is due to flow modification. The polymer stress suppressing the base flow is a typical behaviour of viscoelasticity. Sometimes the flow modification is so severe that it overwhelms the direct effect of the viscoelastic stress (Ramaswamy \& Leal 1999a).

The pressure profile is determined to a large extent by the tangential stress $\tau_{p t}$ due to the polymer (figure $5 c, d$ ). The highly stretched dumbbells near the equator generate 

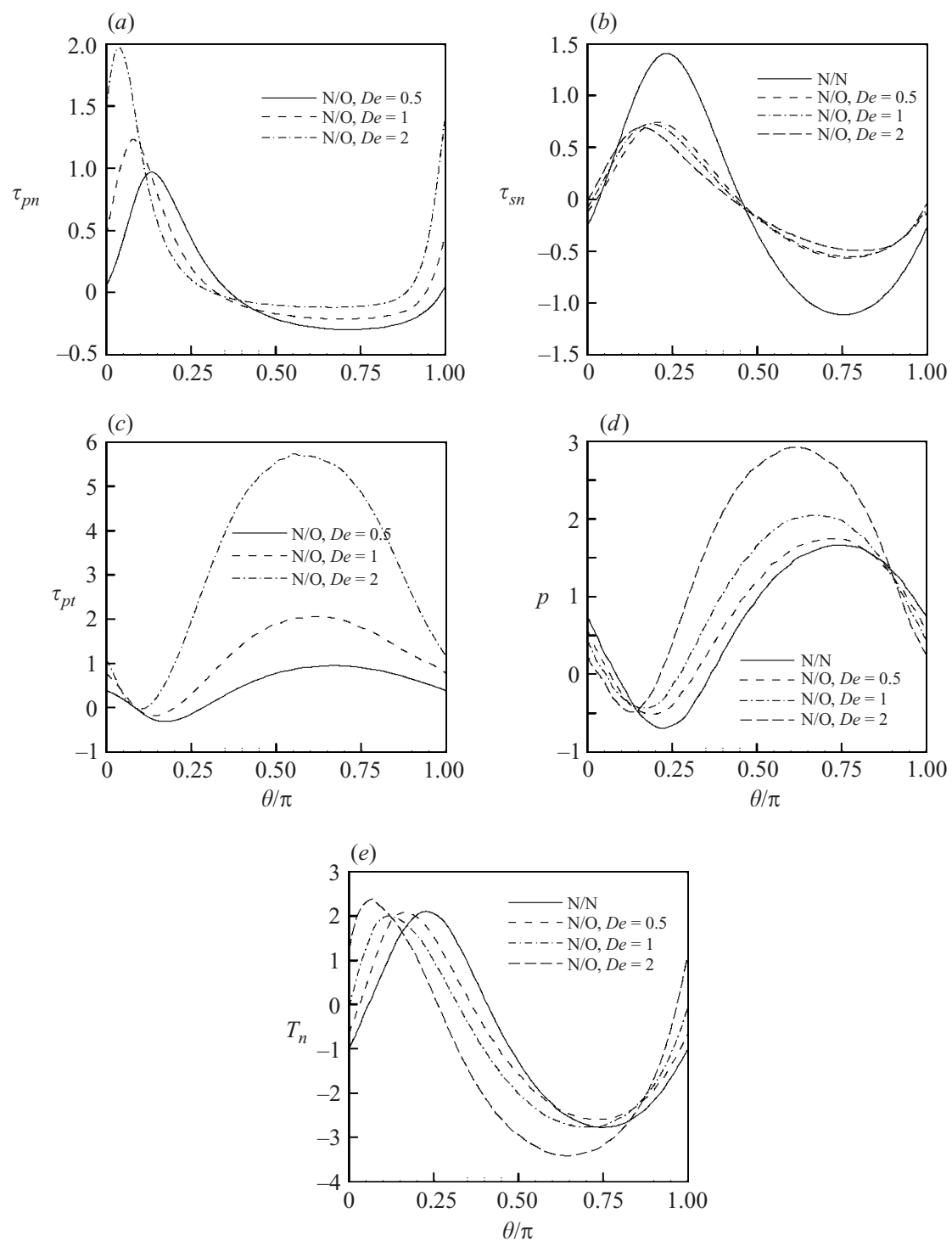

FiguRE 5. Stresses along the outer edge of the interface for N/O at $C a=0.1$. (a) The polymer normal stress $\tau_{p n}=\boldsymbol{n} \cdot \boldsymbol{\tau}_{p} \cdot \boldsymbol{n}, \boldsymbol{n}$ being the outward normal to the interface. ( $b$ ) The viscous normal stress $\tau_{s n}$ due to the solvent in the Oldroyd-B fluid. The $\mathrm{N} / \mathrm{N}$ curve is also shown for comparison. (c) The polymer tangential stress $\tau_{p t} .(d)$ The pressure $p$. (e) The total normal stress $T_{n}=\tau_{p n}+\tau_{s n}-p$.

a large tensile $\tau_{p t}$, which, though not acting directly to deform the drop, induces a very large pressure near the equator much as the hoop-stress causes rod-climbing. Thus, we have identified three effects of viscoelasticity in the matrix: the polymer normal stress rotates the drop toward the flow direction and stretches the drop at the poles; the viscous normal stress is generally reduced by viscoelasticity due to weakening of the flow; the tensile stress due to extended polymer chains produces a high pressure on the drop's equator. The first and third effects tend to enhance drop deformation, while the second tends to reduce drop deformation. The subtle interplay of these effects is illustrated by the total normal stress $T_{n}$ in figure 5(e). 

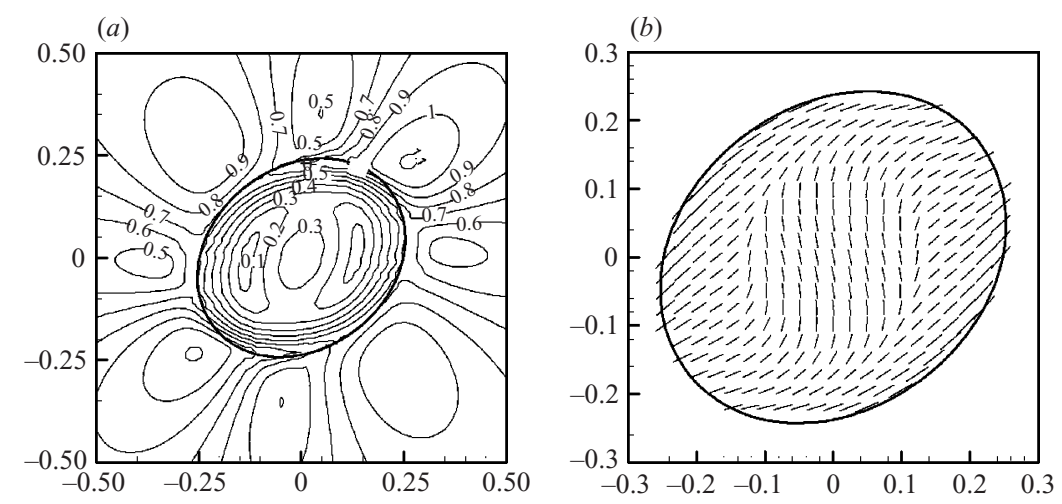

FIgURE 6. (a) Contours of the strain rate $\|\boldsymbol{E}\|=\sqrt{\boldsymbol{E}: \boldsymbol{E}}$, where $\boldsymbol{E}=\left(\nabla \boldsymbol{v}+\nabla \boldsymbol{v}^{\mathrm{T}}\right) / 2$, for $\mathrm{O} / \mathrm{N}$ at $C a=0.1, D e=1$. (b) Predominant orientation of dumbbells inside the drop. The length of the line segments is proportional to $\operatorname{tr}(\boldsymbol{A})$. Note that the line segments represent ensembles of dumbbells; their average orientation is steady although individual dumbbells may be tumbling in the rotational flow.

With $D e$ increasing from 0 to 0.5 , the $T_{n}(\theta)$ profile shifts almost as a whole toward smaller $\theta$, the only distortion to the profile being the reduced magnitude of $T_{n}$ near $\theta \approx 0.6 \pi$. In other words, the compressive force on the equator of the drop is relieved somewhat. Among the components analysed above, this can be attributed to $\tau_{s n}$. Note that $\tau_{p n}$ also exerts less compression there, but the effect is weaker than that of $\tau_{s n}$. Thus, for smaller De, the drop deforms less because the flow modification due to polymer stress diminishes the viscous normal force pushing on the equator of the drop. The pressure $p$ is increased slightly on the equator, but it is insufficient to overcome the deficit in $\tau_{s n}$. As $D e$ exceeds a threshold, approximately 0.5 for $C a=0.1$, the mounting pressure $p$ on the equator becomes the dominant effect. Thus, it is the hoop stress atop the equator that causes the drop deformation $D$ to increase with De at higher De. The polymer stress $\tau_{p n}$ also enhances drop deformation by increasing the stretch on the poles of the drop, but this is a minor effect.

\subsection{An Oldroyd-B drop in a Newtonian matrix $(O / N)$}

For an Oldroyd-B drop deforming in a Newtonian matrix $(\mathrm{O} / \mathrm{N})$, the situation is simpler because the flow modification is minimal. Figure $6(a)$ shows contours of the strain rate for $\mathrm{O} / \mathrm{N}$; they are essentially identical to those for $\mathrm{N} / \mathrm{N}$ at the same $\mathrm{Ca}$, even inside the viscoelastic drop. Outside the drop, the normal force on the interface is due to $\tau_{s n}$ and $p$, and we have verified that they are nearly identical for $\mathrm{N} / \mathrm{N}$ and $\mathrm{O} / \mathrm{N}$. Inside the drop, the flow is highly rotational and the strain rate is small. Thus the viscoelastic stresses inside the drop must be small also. Nevertheless, it must be these viscoelastic stresses that have caused the mild reduction in drop deformation seen in figure 1.

Figure 6(b) gives the distribution of dumbbells inside the Oldroyd-B drop, which may affect the deformation of the drop in two ways. First, at the poles of the drop, the dumbbells are perpendicular to the interface and the polymeric stress tends to pull the interface inward and thus decreases the drop deformation (Pillapakkam \& Singh 2001). Second, $\operatorname{tr}(\boldsymbol{A})$ is maximum at the equator where the dumbbell are nearly parallel to the interface. The resulting strong tension engenders a high pressure at the equator which tends to push the interface outwards. 

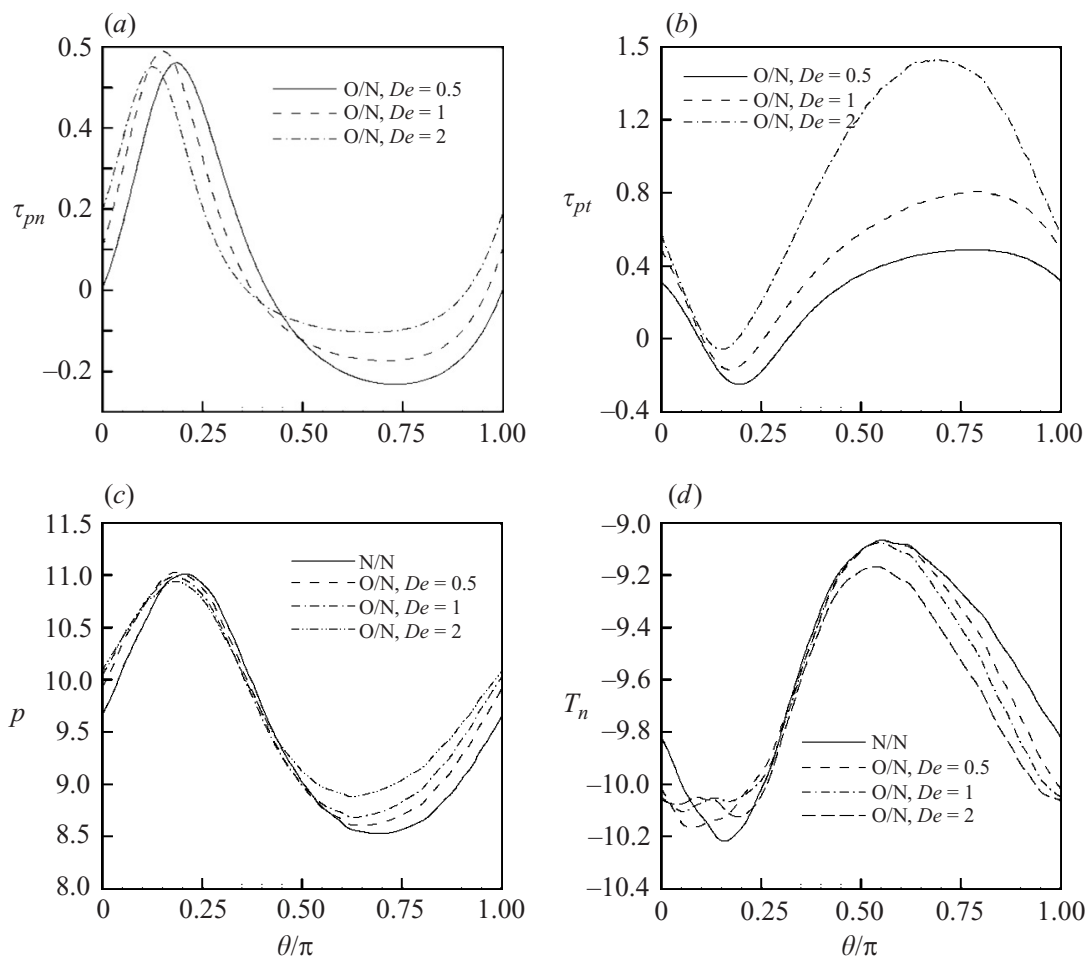

FiguRE 7. Stresses along the inner edge of the interface for $\mathrm{O} / \mathrm{N}$ at $C a=0.1$. ( $a$ ) The polymer normal stress. $(b)$ The polymer tangential stress. (c) The pressure. The higher level of $p$ is a consequence of the Young-Laplace equation. $(d)$ The total normal stress.

Figure 7 gives the stress distributions along the inner edge of the interface. Compared with figure $5, \tau_{p n}$ is much smaller, and varying $D e$ has a much weaker effect on all the stresses plotted. This confirms that viscoelasticity in the drop has less effect on drop deformation than that in the matrix. The distribution of $\tau_{p n}$ is such that it pulls inward on the poles of the drop and pushes mildly outward at the equator (figure $7 a$ ). Thus, it tends to suppress drop deformation. This turns out to be a very weak effect. A more prominent effect is the increase of $\tau_{p t}$ with $D e$ at the equator (figure $7 b$ ). The acceleration of a fluid element just inside the interface is related to $\partial\left(-p+\tau_{p t}\right) / \partial t, t$ being the tangential direction. With little flow modification, an increase in the tensile stress $\tau_{p t}$ due to the polymer is balanced by an increase in $p$, as is evident in figure 7(c). Thus, with increasing $D e, p$ pushes harder outward at the equator. The total normal stress $T_{n}$ largely mirrors the behaviour of $p$. In the end, the decrease of drop deformation $D$ with drop-phase viscoelasticity is caused by the growing outward pressure at the equator of the drop, which is in turn caused by the large viscoelastic tensile stress there. Pillapakkam \& Singh (2001) simulated moreslender drops at larger $C a$, which may not exhibit the large $\tau_{p t}$ at the equator. This may explain their non-monotonic $D \sim D e$ curve for $\mathrm{O} / \mathrm{N}$.

\section{Concluding remarks}

In summary, our analysis is guided by Ramaswamy \& Leal's (1999a) idea that viscoelastic effects come not only from the direct contribution of the non-Newtonian 
stresses, but also from the modification of flow by such stresses. For N/O, it is the coupling of the two factors that gives rise to the non-monotonic $D \sim D e$ curve in figure 1 . For $\mathrm{O} / \mathrm{N}$, there happens to be little flow modification, and we predict a monotonic decrease of $D$ with $D e$.

Since our computations are in two dimensions, quantitative comparison with experiments is difficult. Nevertheless, figure 1 shows reasonable agreement with the experiment of Guido et al. (2003) and the model prediction of Maffettone \& Greco (2004). For smaller $D e$, viscoelasticity in the matrix suppresses deformation more than if it is in the drop, and the effect appears to be stronger in reality than in our simulations. Guido et al.'s (2003) observation of drop rotation is reproduced by our N/O simulations as well (cf. figure 2), and explained by the normal stress $\tau_{p n}$ pulling on the poles of the drop. Perhaps most interestingly, our prediction of a non-monotonic $D \sim D e$ curve for viscoelasticity in the matrix resolves an apparent contradiction among previous experiments at different $D e$. The De range for the upward portion of our curve is consistent with experiments. For instance, Mighri et al. (1998) reported a $100 \%$ increase in $D$ due to matrix elasticity at $D e \approx 2.6$ and $\mathrm{Ca}=0.2$.

The two-dimensionality handicaps this study. In extreme cases such as capillary breakup of a thread, disregarding the curvature in the third dimension leads to qualitatively different predictions. In most cases, however, the difference is quantitative. An example is an extended drop retracting at a slightly different speed in two dimensions than in three. The comparison with three-dimensional results in figure 1 lends some confidence to our two-dimensional simulations. In general, extending a two-dimensional computation to three dimensions involves technical rather than conceptual difficulties. In our spectral implementation, using the regular mesh in three dimensions would produce too large a computational task. In recent years, a few three-dimensional simulations have appeared. But they tend to use coarse grids with insufficient resolution (Khayat 2000) and generate numerical results, for the same physical problem, that do not agree. The key to reliable three-dimensional simulations seems to lie in adaptive meshing. Work is under way for a finite-element method with adaptive meshing for three-dimensional flows in complex geometries.

Acknowledgment is made to the Donors of the Petroleum Research Fund, administered by the American Chemical Society, for partial support of this research. J.J.F. was also supported by the NSF (CTS-0229298, CTS-9984402), the NSERC and the Canada Research Chair program, and the NNSF of China (No. 20174024 and No. 20490220). J. S. was supported by the NSF (DMS-0074283, DMS-0311915). C. L. was supported by the NSF (DMS-0405850).

\section{REFERENCES}

Bird, R. B., Curtiss, D. F., Armstrong, R. C. \& Hassager, O. 1987 Dynamics of Polymeric Liquids, Vol. 2. Kinetic Theory. Wiley.

Elmendorp, J. J. \& MaAlcke, R. J. 1985 A study on polymer blending microrheology: Part I. Polymer Engng Sci. 25, 1041-1047.

Feng, J. J., Liu, C., Shen, J. \& Yue, P. 2005 An energetic variational formulation with phase field methods for interfacial dynamics of complex fluids: advantages and challenges. In Modeling of Soft Matter (ed. M.-C. T. Calderer \& E. Terentjev). IMA Volumes in Mathematics and its Applications. Springer.

Flumerfelt, R. W. 1972 Drop breakup in simple shear fields of viscoelastic fluids. Ind. Engng Chem. Fundam. 11, 312-318. 
Greco, F. 2002 Drop deformation for non-Newtonian fluids in slow flows. J. Non-Newtonian Fluid Mech. 107, 111-131.

Guido, S., Simeone, M. \& Greco, F. 2003 Deformation of a Newtonian drop in a viscoelastic matrix under steady shear flow. Experimental validation of slow flow theory. J. Non-Newtonian Fluid Mech. 114, 65-82.

Hooper, R. W., de Almeida, V. F., Macosko, C. W. \& Derby, J. J. 2001 Transient polymeric drop extension and retraction in uniaxial extensional flows. J. Non-Newtonian Fluid Mech. 98, $141-168$.

JACQMin, D. 1999 Calculation of two-phase Navier-Stokes flows using phase-field modelling. J. Comput. Phys. 155, 96-127.

Khayat, R. E. 2000 Three-dimensional boundary element analysis of drop deformation in confined flow for Newtonian and viscoelastic systems. Intl J. Numer. Methods Fluids 34, 241-275.

Khismatullin, D., Renardy, Y. \& RenARDy, M. 2005 Development and implementation of VOF-PROST for 3D viscoelastic liquid-liquid simulations. J. Non-Newtonian Fluid Mech. (submitted).

Lerdwijitjarud, W., Sirivat, A. \& Larson, R. G. 2004 Influence of dispersed-phase elasticity on steady-state deformation and breakup of droplets in simple shearing flow of immiscible polymer blends. J. Rheol. 48, 843-862.

Levitt, L., Macosko, C. W. \& Pearson, S. D. 1996 Influence of normal stress difference on polymer drop deformation. Polymer Engng Sci. 36, 1647-1655.

Maffettone, P. L. \& Greco, F. 2004 Ellipsoidal drop model for single drop dynamics with non-Newtonian fluids. J. Rheol. 48, 83-100.

Mighri, F., Ajui, A. \& CARreau, P. J. 1997 Influence of elastic properties on drop deformation in elongational flow. J. Rheol. 41, 1183-1201.

Mighri, F., Carreau, P. J. \& AJji, A. 1998 Influence of elastic properties on drop deformation and breakup in shear flow. J. Rheol. 42, 1477-1490.

Pillapakkam, S. B. \& Singh, P. 2001 A level-set method for computing solutions to viscoelastic two-phase flow. J. Comput. Phys. 174, 552-578.

Rallison, J. M. 1984 The deformation of small viscous drops and bubbles in shear flows. Annu. Rev. Fluid Mech. 16, 45-66.

Ramaswamy, S. \& Leal, L. G. 1999a The deformation of a viscoelastic drop subjected to steady uniaxial extensional flow of a Newtonian fluid. J. Non-Newtonian Fluid Mech. 85, 127-163.

Ramaswamy, S. \& Leal, L. G. $1999 b$ The deformation of a Newtonian drop in the uniaxial extensional flow of a viscoelastic liquid J. Non-Newtonian Fluid Mech. 88, 149-172.

Stone, H. A. 1994 Dynamics of drop deformation and breakup in viscous fluids. Annu. Rev. Fluid Mech. 26, 65-102.

TAYlor, G. I. 1932 The viscosity of a fluid containing small drops of another fluid. Proc. R. Soc. Lond. A 138 41-48

Tretheway, D. C. \& Leal, L. G. 2001 Deformation and relaxation of Newtonian drops in planar extensional flow of a Boger fluid. J. Non-Newtonian Fluid Mech. 99, 81-108.

Tucker, C. L. \& Moldenaers, P. 2002 Microstructural evolution in polymer blends. Annu. Rev. Fluid Mech. 34, 177-210.

VANOEne, H. 1972 Models of dispersion of viscoelastic fluids in flow. J. Colloid Interface Sci. 40, 448-467.

Yu, W., Zhou, C. \& BousminA, M. 2005 Theory of morphology evolution in mixtures of viscoelastic immiscible components. J. Rheol. 49, 215-236.

Yue, P., Feng, J. J., Liu, C. \& Shen, J. 2004 A diffuse-interface method for simulating two-phase flows of complex fluids. J. Fluid Mech. 515, 293-317. 